\title{
Long non-coding RNA UCA1 promotes papillary thyroid cancer cell proliferation via miR-204-mediated BRD4 activation
}

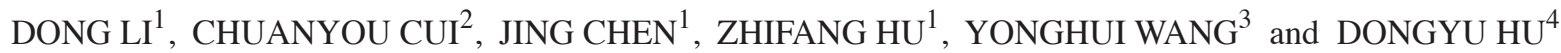 \\ ${ }^{1}$ Department of Endocrinology 1, Affiliated Hospital of Jining Medical University, Jining, Shandong 272029; \\ ${ }^{2}$ Department of Breast, Thyroid and Hernia Surgery, Liaocheng People's Hospital, Liaocheng, Shandong 252000; \\ ${ }^{3}$ Department of Breast Surgery, Weifang People's Hospital, Weifang, Shandong 261041; ${ }^{4}$ Department of Oncology, \\ Affiliated Hospital of Jining Medical University, Jining, Shandong 272029, P.R. China
}

Received January 8, 2018; Accepted June 19, 2018

DOI: $10.3892 / \mathrm{mmr} .2018 .9246$

\begin{abstract}
Long non-coding RNA (lncRNA) urothelial carcinoma-associated 1 (UCA1) has been used in tumor development and progression in many types of cancer. However, the function and mechanism underlying the action of UCA1 in papillary thyroid cancer (PTC) remains unclear. Therefore, these topics were investigated in the present study by in vitro and in vivo experiments. It was demonstrated that the expression level of UCA1 was more significantly upregulated in PTC cell lines and tissues when compared with the immortal human thyroid follicular cell line and adjacent normal tissues, respectively. UCA1 knockdown significantly inhibited PTC cell viability, colony formation and the bromodomain containing 4 (BRD4) expression level in vitro, and retarded PTC tumor growth in vivo. In the previous study, microRNA (miR)-204 inhibited thyroid cancer progression and was regulated by UCA1 in other types of cancer. In addition, by conducting dual luciferase reporter assays, it was confirmed that miR-204 directly binds to UCA1 and the 3'-untranslated region of BRD4. Furthermore, UCA1 competed with BRD4 for miR-204 binding. miR-204 knockdown enhanced BRD4 expression, which can be partially restored by short hairpin-UCA1. The results of the present study illustrated that UCA1 promotes PTC progression by acting as a competing endogenous RNA by sponging miR-204. In conclusion, UCA1 may be regarded as an oncogenic lncRNA, promoting PTC cell proliferation, and be a potential target for human PTC treatment.
\end{abstract}

\section{Introduction}

Thyroid cancer is the most common tumour of the endocrine system, and its incidence rate has dramatically increased

Correspondence to: Professor Dongyu Hu, Department of Oncology, Affiliated Hospital of Jining Medical University, Building 5, 89 Guhuai Road, Jining, Shandong 272029, P.R. China

E-mail: hdy1215@126.com

Key words: papillary thyroid cancer, proliferation, urothelial carcinoma-associated 1, microRNA-204, bromodomain containing 4 over the past several decades (1). Thyroid cancer incidence is rapidly increasing in the USA; in 2017, its estimated annual diagnosis rate and annual mortality rate were 56,870 people and 2,010 cases, respectively (2). Papillary thyroid carcinoma (PTC) accounts for the majority of thyroid cancers and generally exhibits favourable prognosis (3). However, the recurrence rate is approximately $15 \%$ in 3 years (4). Recurrent PTC has limited therapeutic options and poor prognosis, and this disease is currently the leading cause of death in patients with PTC. Therefore, our understanding of the mechanisms underlying the pathogenesis of PTC should be improved, and further effective therapeutic approaches should be developed.

Long non-coding RNAs (lncRNAs) are a diverse class of RNAs with a length of more than 200 nucleotides that do not encode proteins (5). Genome-wide transcriptional analysis has revealed the high prevalence of lncRNAs in all transcripts (5). lncRNAs are important epigenetic modulators with sensory, guiding and scaffolding capacities (6). Thus, lncRNAs have gained increasing attention as a critical regulator of normal physiology and disease development, particularly in proliferation and migration (7). IncRNA expression is dysregulated in many diseases affecting different tissue types (7). lncRNAs can be detected in various human body fluids, such as serum and plasma, suggesting that lncRNAs may serve as new biomarkers for disease diagnosis and prognosis without invasive procedures (8).

Urothelial carcinoma-associated 1 (UCA1) is a lncRNA that shows potential as a biomarker for cancer. The UCA1 gene is located at chromosome 19p-13.12 and dysregulated in breast cancer, oesophageal cancer, gastric cancer and bladder cancer (9-13). UCA1 depletion can attenuate the migratory ability of various cancers (9-13), whereas UCA1 overexpression can enhance ovarian cancer cell migration and invasion (14), suggesting that UCA1 functions as a pivotal regulator of cellular migration and invasion. Nevertheless, up to now, there is no relevant report about the relationship between UCA1 and the progression of PTC. Therefore, the role and underlying mechanism of UCA1 in PTC should be elucidated.

Previous studies have showed that miR-204 plays a protective role by inhibiting thyroid cancer cell proliferation and may identify new targets for anti-cancer treatment $(15,16)$. Moreover, in the recent studies has showed that UCA1 can 
directly regulated the expression of miR-204 in prostate cancer, colorectal cancer and oesophageal cancer $(9-11,17)$. However, the correlation of UCA1 and miR-204 remains elusive in PTC. Therefore, there is a vital necessity to illustrate the relationship of UCA1 and miR-204 in PTC.

In this study, we reported an interaction between UCA1 and miR-204, which regulates PTC cell growth by targeting BRD4. Our study demonstrated that the UCA1/miR-204/BRD4 signalling pathway might be a novel therapeutic target for patients with PTC.

\section{Materials and methods}

Cell culture and tissue collections. The human PTC cell lines (TPC-1 and BCPAP) and immortal human thyroid follicular cell line (Nthy-ori3-1) were obtained from the Cell Bank of China (Shanghai,China). These cell lines were authenticated via short-tandem repeat profiling performed by BMR Genomics. BCPAP usually known as a PTC cell line is derived from a poorly differentiated PTC, however, recent study has showed that the mRNA expression profile of BCPAP were closer to anaplastic thyroid cancer cells (18). In this study, BCPAP serves as poorly differentiated papillary thyroid cancer cell line to further confirm the experiments results of TPC-1 and not affect the outcomes of this study. The cells were cultured in DMEM (HyClone; GE Healthcare Life Sciences, Shanghai, China) supplemented with $10 \%$ foetal bovine serum (HyClone; GE Healthcare Life Sciences) in a humidified atmosphere with $5 \% \mathrm{CO}_{2}$ and humidified atmosphere of $95 \%$ at $37^{\circ} \mathrm{C}$. Human PTC specimens and their adjacent normal thyroid tissues were obtained from 30 patients who had PTC and who had undergone surgery at the Jining Medical University. All tissue samples were immediately frozen in liquid $\mathrm{N}$ and stored at $-80^{\circ} \mathrm{C}$ until RNA extraction. Written informed consent was obtained from each patient, and the study protocol and consent procedures were approved by the Ethics Committee of the Jining Medical University (Shandong, China).

Cell transfection. LV3 (H1/GFP\&Puro) vector was synthesized for Lv-shRNAUCA1 (Guangzhou RiboBio Co., Ltd., Guangzhou, China). A nontarget scrambled oligonucleotide served as the negative control. The shRNA sequences used in the present study were as follows: shUCA1, 5'-GCCACCUACAUUAAAGCU AdTdT-3' and shcontrol, 5'-CAGUACUUUUGUGUAGUACAA-3'. miR-204 mimic (miR10000265-1-5), miR-NC (miR01201-1-5), inhibitor (miR20022693-1-5) and anti-NC (miR02201-1-5) were obtained from Guangzhou RiboBio Co., Ltd.. Transfection was performed by using Lipofectamine 2000 reagent (Invitrogen; Thermo Fisher Scientific, Inc., Waltham, MA, USA) according to the manufacturer's protocols.

Western blot analysis. Equal amounts of proteins (30 $\mu \mathrm{g})$ from the lysates of the cells were subjected to electrophoresis through a $10 \%$ SDS PAGE (Beyotime Institute of Biotechnology, Haimen, China) at $80 \mathrm{~V}$ for $30 \mathrm{~min}$ and at $100 \mathrm{~V}$ for $1.5 \mathrm{~h}$. The proteins were then transferred onto polyvinylidene difluoride membranes. After blocking in 5\% skimmed milk, the membranes were then incubated with the following diluted primary antibodies: Rabbit polyclonal BRD4 (ab84776, 1:500,
Abcam, Cambridge, MA, USA), mouse monoclonal GAPDH (AF0006, 1:1,000, Beyotime Institute of Biotechnology) overnight at $4{ }^{\circ} \mathrm{C}$ and peroxidase-coupled secondary antibody (1:2,000; cat. no. A0216; Beyotime Institute of Biotechnology) at room temperature for $2 \mathrm{~h}$. Specific bands were visualised on an autoradiographic film using an enhanced chemiluminescence reagent (Nanjing KeyGen Biotech Co., Ltd., Nanjing, China).

Reverse transcription-quantitative polymerase chain reaction (RT-qPCR). Total RNA from cells and tissues was isolated with a TRIzol reagent (Invitrogen; Thermo Fisher Scientific, Inc.) according to the manufacturer's instructions. First-strand cDNA was generated by reverse transcribing the total cellular RNA with M-MLV reverse transcriptase (Promega Corporation, Madison, WI, USA). The SYBR Premix Ex Taq $^{\mathrm{TM}}$ kit (Takara Bio, Inc., Otsu, Japan) was used according to the manufacturer's instructions, and RT-qPCR was performed and analysed by using the iQ5 detection system (Bio-Rad Laboratories, Inc., Hercules, CA, USA). The GAPDH served as the internal control for detection. The qPCR conditions were applied for detecting mRNAs: $95^{\circ} \mathrm{C}$ for $30 \mathrm{sec}$, followed by 40 cycles of $95^{\circ} \mathrm{C}$ for $30 \mathrm{sec}, 60^{\circ} \mathrm{C}$ for $30 \mathrm{sec}$ and $72^{\circ} \mathrm{C}$ for $30 \mathrm{sec}$. Relative mRNA expression was analyzed as the inverse $\log$ of $\Delta \Delta \mathrm{Cq}$ and was normalized to the reference (19).

UCA1: Forward 5'-TTTGCCAGCCTCAGCTTAAT-3', Reverse 5'-TTGTCCCCATTTTCCATCAT-3'; miR-204 Forward 5'-GTCCCTGTGTCATCCT-3', Reverse 5'-CAGTGCAGGGTCCGAGGTAT-3'; U6 Forward 5'-TGC GGGTGCTCGCTTCGCAGC-3', Reverse 5'-CCAGTGC AGGGTCCGAGGT-3'; BRD4 Forward 5'-CATGGACA TGAGCACAATCA-3', Reverse 5'-TCATGGTCAGG AGGGTTGTA-3'; GAPDH Forward 5'-CTGACCTGCCG TCTAGAAA-3', Reverse 5'-GTGGTGTGACTTAGAG GGG-3'.

Cell viability assay. Cell viability was examined using CCK-8 assay. Briefly, TPC-1 and BCPAP cells transfected with either shUCA1 or shcontrol were plated onto 96 -well plates with 3,000 cells/well. After culturing at an indicated time $(0,24$, 48 and $72 \mathrm{~h}$ ), $10 \mu \mathrm{l}$ of CCK-8 solution was added into each well at $37^{\circ} \mathrm{C}$. After $3 \mathrm{~h}$, absorbance was determined using a microplate spectrophotometer at a wavelength of $450 \mathrm{~nm}$.

Colony formation assay. The TPC-1 and BCPAP cells transfected with either shUCA1 or shcontrol (500 cells/well) were placed into 6-well plates. After 1 week, the colonies were fixed with methanol and stained with $0.1 \%$ crystal violet for $20 \mathrm{~min}$, and the images of the stained colonies were captured by using a CKX41 light microscope (Thermo Labsystems, Vantaa, Finland). The number of the colonies was counted by using the images.

Migration and invasion assay. Migration and invasion ability was examined by using Transwell assay. For the invasion assay, the upper sides of the filters were coated with $50 \mu 1$ of Matrigel (BD Biosciences, Franklin Lakes, NJ, USA). Indicated cells were plated at a density of $5 \times 10^{4}$ per well in the upper chamber without serum. Cells were incubated for $12 \mathrm{~h}$ for the migration assay and $24 \mathrm{~h}$ for the invasion assay. After 

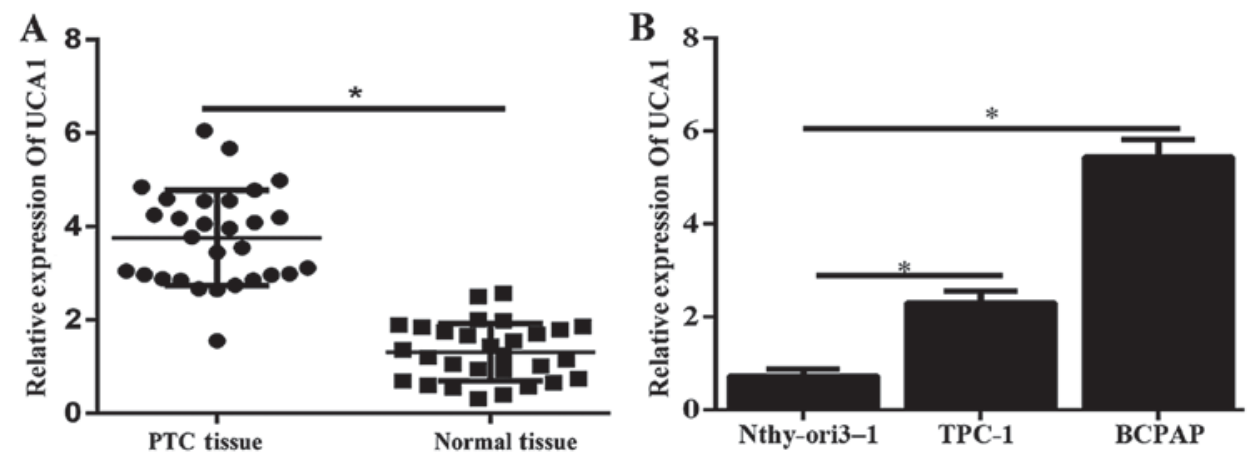

Figure 1. UCA1 expression is upregulated in PTC tissues and cell lines. (A) Relative UCA1 expression was examined by RT-qPCR in 30 pairs of thyroid cancer specimens and adjacent normal tissues. GAPDH served as the internal control. (B) The expression level of UCA1 was also examined in PTC cell lines (TPC-1 and BCPAP) and in human immortal follicular thyroid cell Nthy-ori3-1 via RT-qPCR. GAPDH was used as internal control. "P $<0.05$, as indicated. UCA1, urothelial carcinoma-associated 1; PTC, papillary thyroid cancer; RT-qPCR, reverse transcription-quantitative polymerase chain reaction.

the cells were incubated at the exact time at $37^{\circ} \mathrm{C}$, the inserts were washed with PBS, and cells on the upper surface of the insert were removed with a cotton swab. Cells adhering to the lower surface were fixed with $4 \%$ formaldehyde for $20 \mathrm{~min}$, stained with $0.1 \%$ crystal violet solution and imaged using a CKX41 light microscope.

Dual luciferase reporter assay. TPC-1 and BCPAP cells cultured in 24-well plates were cotransfected with luciferase reporter plasmids (wt-BRD4, mut-BRD4 containing miR-204 binding site, wt-UCA1 and mut-UCA1 containing miR-204 binding site) and miRNA mimics or inhibitor. After $48 \mathrm{~h}$, luciferase activity was measured via dual-luciferase reporter assay system according to the manufacturer's instruction (Promega Corporation).

In vivo assay. All the procedures were carried out in accordance to the Guide for the Care and Use of Laboratory Animals issued by the Institutional Animal Care and Use Committee of the Jining Medical University (Jining, China). Five-week-old female BALB/c nude mice were purchased from the Yangzhou Laboratory Animal Centre and maintained in a SPF environment. Subcutaneous tumour xenografting was performed by subcutaneously injecting mice with $100 \mu \mathrm{l}$ of PBS containing $5 \times 10^{5}$ BCPAP cells that had been transfected with either shUCA1 or shcontrol. Tumour volume $\left(\mathrm{mm}^{3}\right)$ was calculated every 7 days by using the following equation: $\mathrm{V}=0.5 \mathrm{x}$ length $\mathrm{x}$ width $^{2}$. After 42 days, the mice were euthanized, and the tumors were isolated, weighed, photographed and then processed for further study.

Immunohistochemistry. Paraffin-embedded sections of tumor tissue ( $4 \mu \mathrm{m}$ thick) were deparaffinized in xylene, rehydrated via graded alcohol solutions, blocked in methanol containing $3 \%$ hydrogen peroxide for $10 \mathrm{~min}$ at room temperature, and then incubated with mouse BRD4 antibody (ab84776, 1:500; Abcam, Cambridge, MA, USA) at $4^{\circ} \mathrm{C}$ overnight. Following rinsing with $\mathrm{PBS}$ solution, biotinylated goat anti-rabbit serum IgG (ab6785, 1:2,000; Abcam, Cambridge, MA, USA) was used as secondary antibodies and streptavidin peroxidase complex reagent were applied for $1 \mathrm{~h}$ at room temperature. Finally, the sections were incubated in a 3,3'-diaminobenzidine solution at room temperature for $10 \mathrm{~min}$ and then counterstained with hematoxylin for $3 \mathrm{~min}$ at room temperature. Ten randomly selected visual fields per section were examined under a light microscope to evaluate the BRD4 expression.

Statistical analysis. All experiments were performed three times. The data were presented as the mean \pm standard deviation. Differences between two groups were assessed using two-tailed Student's t-test. Data from $>2$ groups were analyzed using one way analysis of variance with the post hoc Tukey's test. Statistical analyses were performed using SPSS version 13.0 (SPSS, Inc., Chicago, IL, USA). Differences were considered statistically significant when $\mathrm{P}<0.05$.

\section{Results}

UCA1 is upregulated in PTC tissues and cell lines. To evaluate the role of UCA1 in PTC, we first detected the expression level of UCA1 in 30 pairs of human PTC specimens and corresponding adjacent non-tumor tissues were determined by using qPCR. Results illustrated that the expression of UCA1 was significantly higher in tumor tissues (Fig. 1A). Furthermore, the expression level of UCA1 in PTC cell lines (TPC-1 and BCPAP) was significantly enhanced compared to that in the human immortal follicular thyroid cell Nthy-ori3-1 (Fig. 1B). Therefore, UCA1 might play a critical role in the progression of PTC.

UCA1 silencing decreased cell viability, proliferation in PTC cell lines. UCA1 knockdown was achieved by transfection with shUCA1 in BCPAP and TPC-1, as verified via qPCR assays (Fig. 2A and B). The CCK-8 results showed that the cell viability of BCPAP and TPC-1 cells was significantly decreased after UCA1 knockdown (Fig. 2C and D). In addition, the colony formation assay results illustrated that the proliferation of UCA1 silencing cells was remarkably decreased (Fig. 2E and F). The Transwell assay illustrated that the migration and invasion of UCA1 silencing cells was significantly decreased (Fig. 2G and H).

Previous study has showed that BRD4 is involved in the progression of PTC (20). BRD4 silencing decreased the tumor viability, proliferation in vitro and in vivo (20). Here we also monitored the protein levels of BRD4 in BCPAP and TPC-1 cells, in response to UCA1 silencing. The western blot 
A

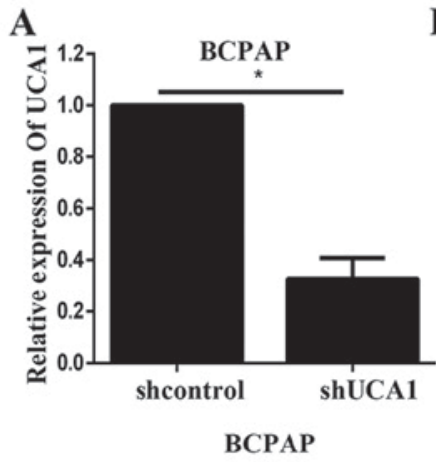

$\mathbf{E}$

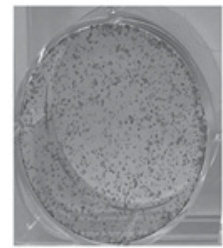

shcontrol

shUCA1
B
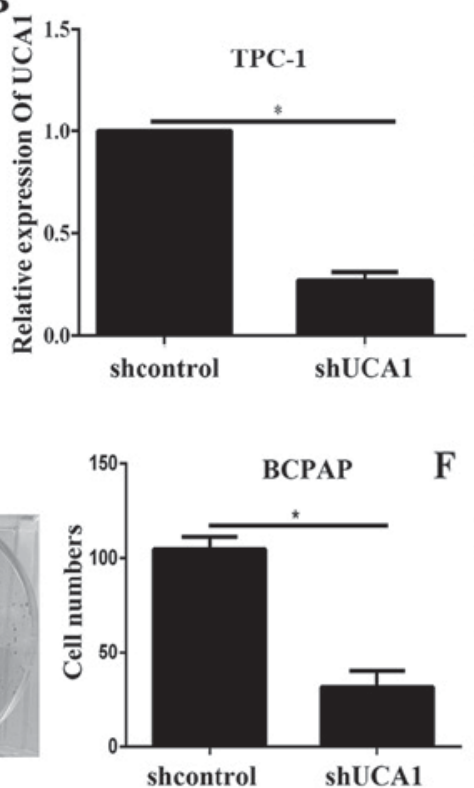

C

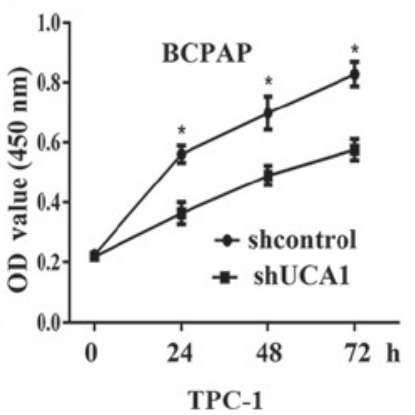

D
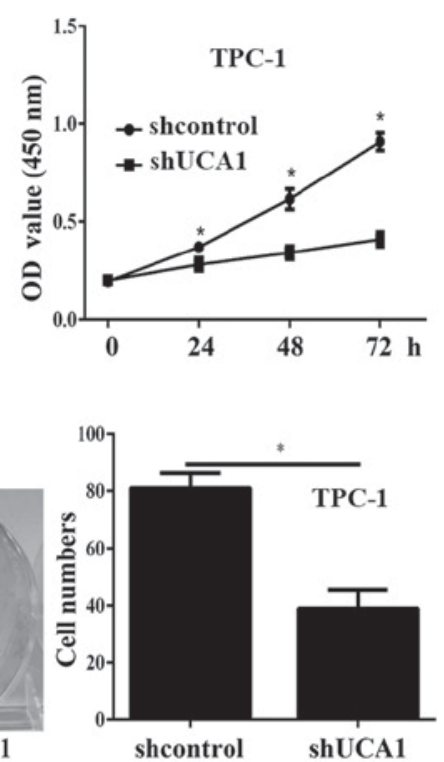

G

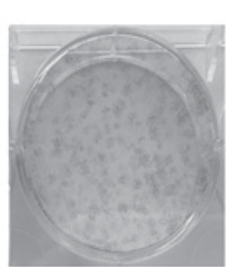

shcontrol

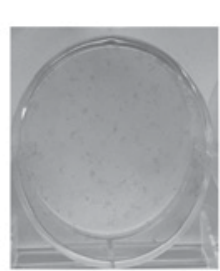

shUCA1

shUCA1
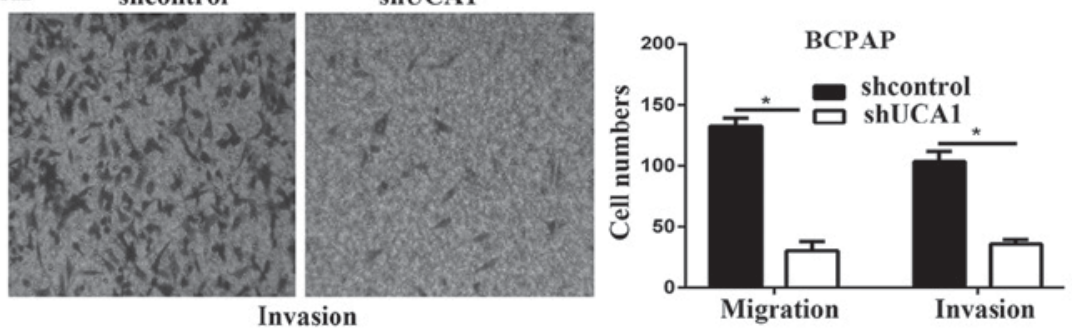

Migration

Invasion

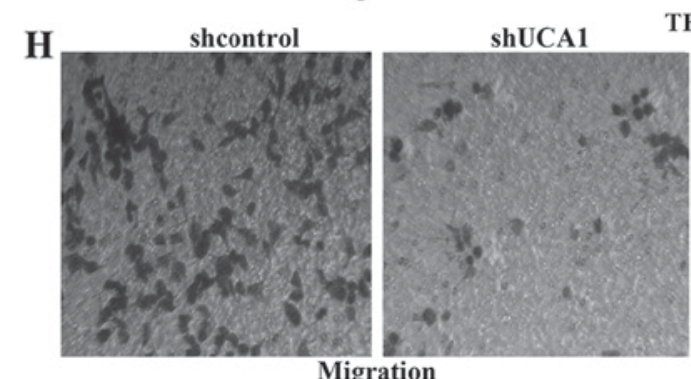

TPC-1
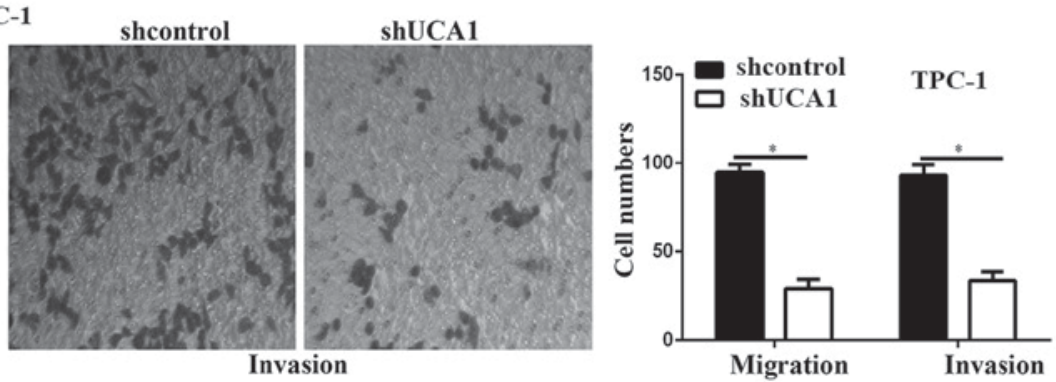

Figure 2. UCA1 promotes PTC cell proliferation. (A) BCPAP and (B) TPC-1 cells were transfected with shUCA1 and shcontrol, and the expression of UCA1 was detected by reverse transcription-quantitative polymerase chain reaction. (C-F) Cell viability and proliferation was separately assessed by (C and D) Cell Counting Kit-8 and (E and F) colony formation assays. ( $\mathrm{G}$ and $\mathrm{H}$ ) The migration and invasion of (G) BCPAP and (H) TPC-1 cells was examined via Transwell assay (magnification, x100). "P<0.05 vs. shcontrol/as indicated. UCA1, urothelial carcinoma-associated 1; PTC, papillary thyroid cancer; sh-, short hairpin RNA.

and qPCR results showed that UCA1 silencing significantly decreased the expression level of BRD4 (Fig. 3A-D). These results illustrated that UCA1 might enhance PTC progression via the activation of BRD4.

miR-204 could negatively regulate BRD4 expression. To discover the underlying mechanism by which UCA1 regulated PTC progression, TargetScan (http://www.targetscan. org/vert_71/) was used. BRD4 was predicted to be a target of miR-204. miR-204 has been demonstrated to serve as tumor suppressor gene in some kinds of cancer including thyroid cancer $(15,16)$. Moreover, recent studies have showed that UCA1 can directly regulate the expression of miR-204 in some types of cancer $(9,11,17)$. BCPAP and TPC- 1 cells were transfected with miR-204 mimic, miR-NC, miR-204 inhibitor or NC-inhibitor respectively. The expression level of miR-204 was examined via qPCR (Fig. 4A and B). The results showed that the mRNA expression of BRD4 was significantly decreased by miR-204 overexpression while enhanced by miR-204 inhibition (Fig. 4C and D). The protein expression levels of BRD4 in BCPAP and TPC-1 cell lines in response to miR-204 change were then examined by using Western blot. Results showed that the protein expression of BRD4 was decreased by miR-204 mimic while increased by miR-204 inhibitor (Fig. 4E and F). These data showed that miR-204 could negatively regulate BRD4 expression in PTC cell lines.

UCAl competed with BRD4 for miR-204 binding. We demonstrated that miR-204 negatively regulated BRD4 expression; then we illustrated the correlation between UCA1 and miR-204 

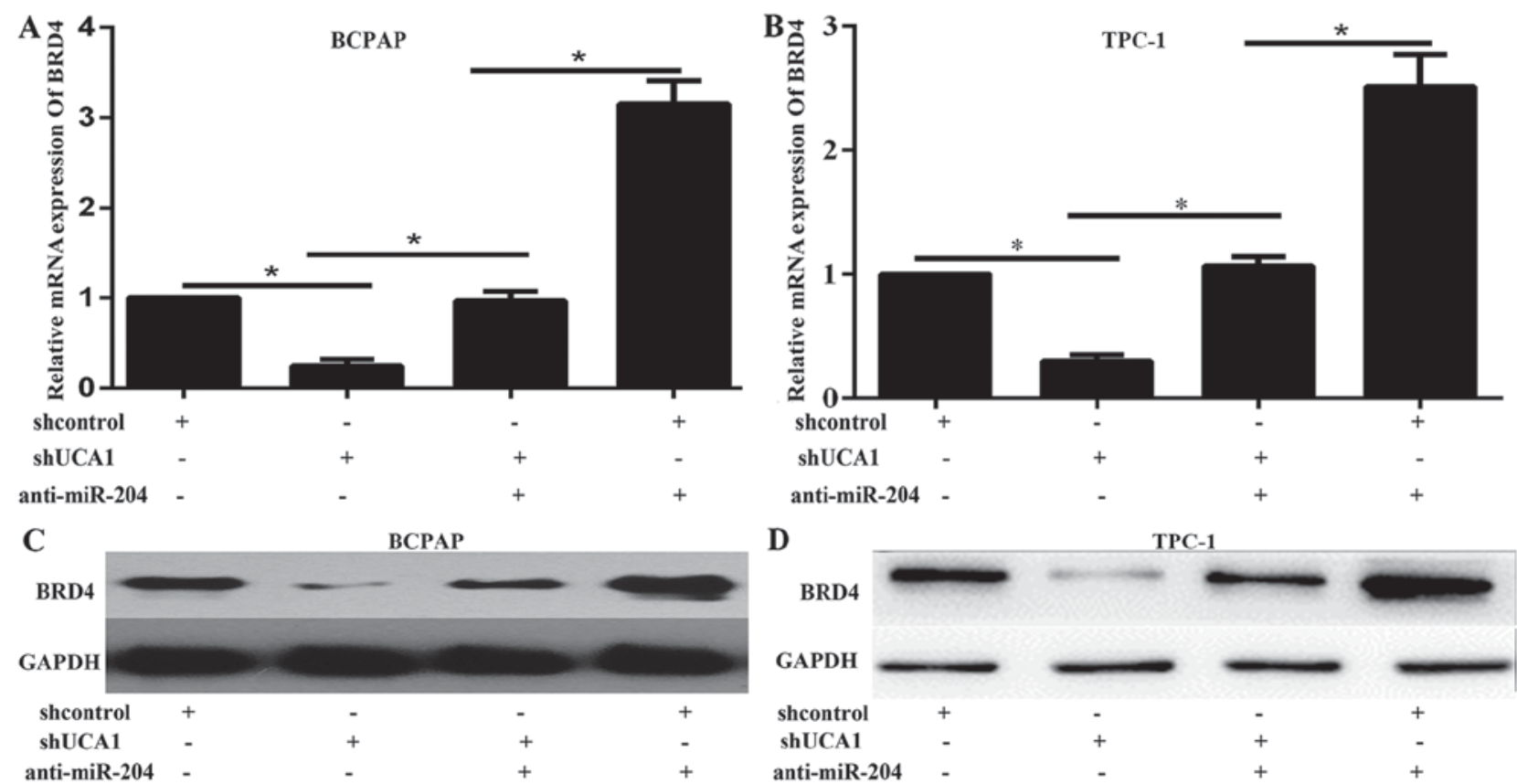

Figure 3. UCA1 regulates BRD4 expression via miR-204. BCPAP and TPC-1 cells were co-transfected with shUCA1 and miR-204 inhibitor (anti- miR-204); the (A and B) mRNA and (C and D) protein levels of BRD4 were then examined via reverse transcription-quantitative polymerase chain reaction and western blot assays, respectively. "P<0.05, as indicated. UCA1, urothelial carcinoma-associated 1; sh-, short hairpin RNA; BRD4, bromodomain containing 4; miR, microRNA.
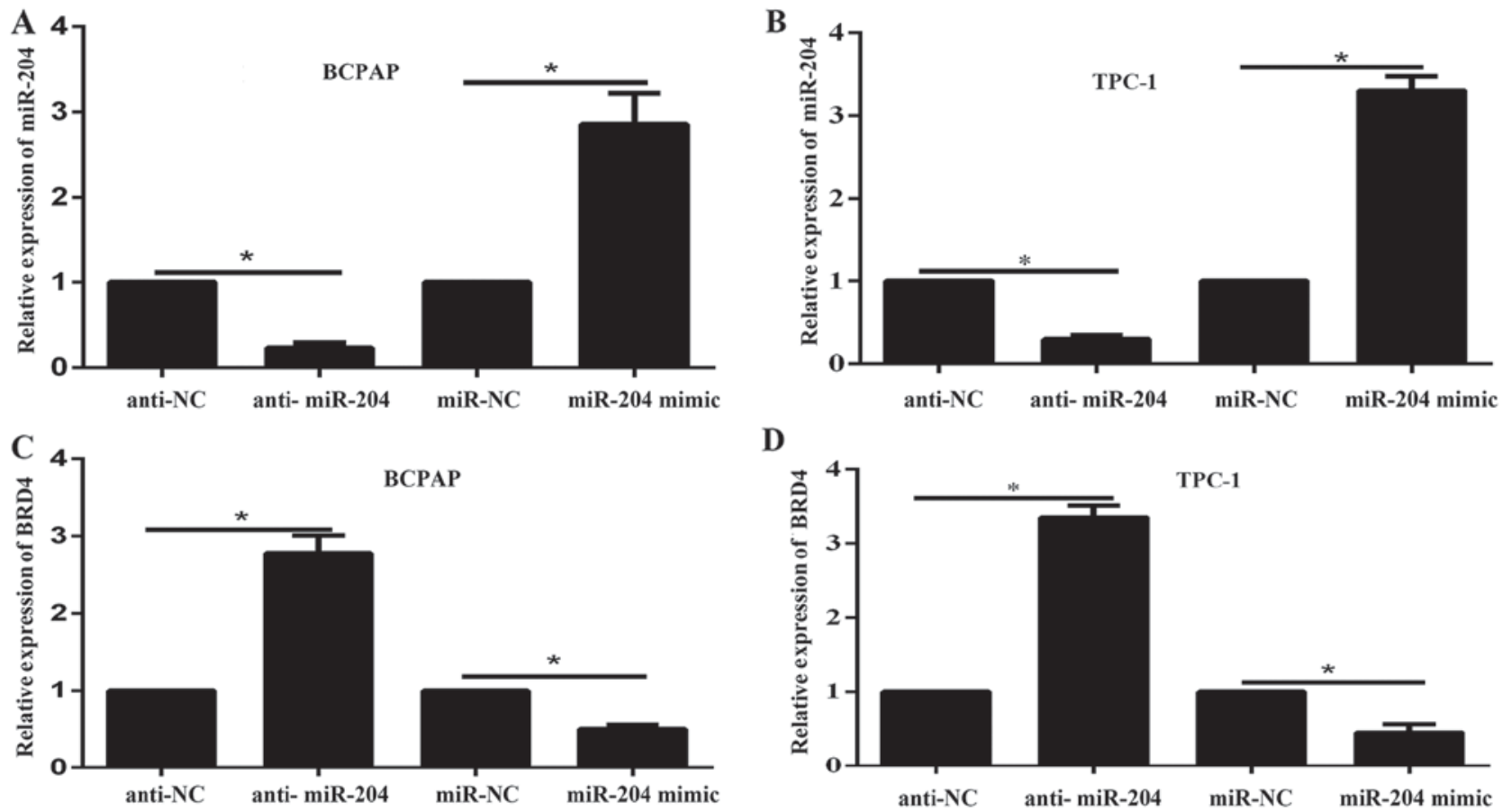

D
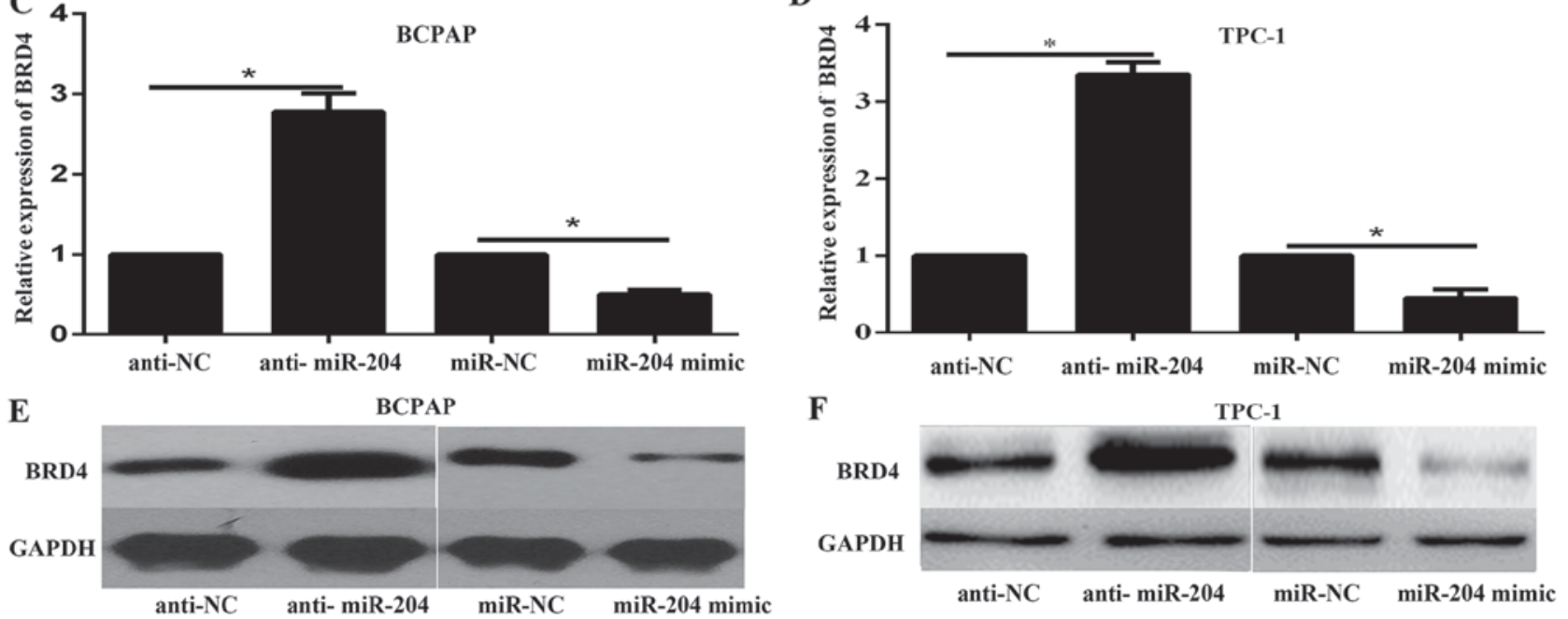

Figure 4. miR-204 negatively regulates BRD4 expression. (A and B) Relative expression levels of miR-204 were determined in thyroid cancer cells transfected with miR-204 mimic, miR-NC, miR-204 inhibitor or NC-inhibitor respectively by RT-qPCR. (C and D) The mRNA expression levels of BRD4 in (C) BCPAP and (D) TPC-1 cells in response to miR-204 expression alteration were also determined by RT-qPCR. (E and F) The protein level of BRD4 in (E) BCPAP and (F) TPC-1 cells in response to miR-204 expression alteration were determined via western blot assays. "P<0.05, as indicated. UCA1, urothelial carcinoma-associated 1; sh-, short hairpin RNA; BRD4, bromodomain containing 4; miR, microRNA; NC, negative control; RT-qPCR, reverse transcription-quantitative polymerase chain reaction. 

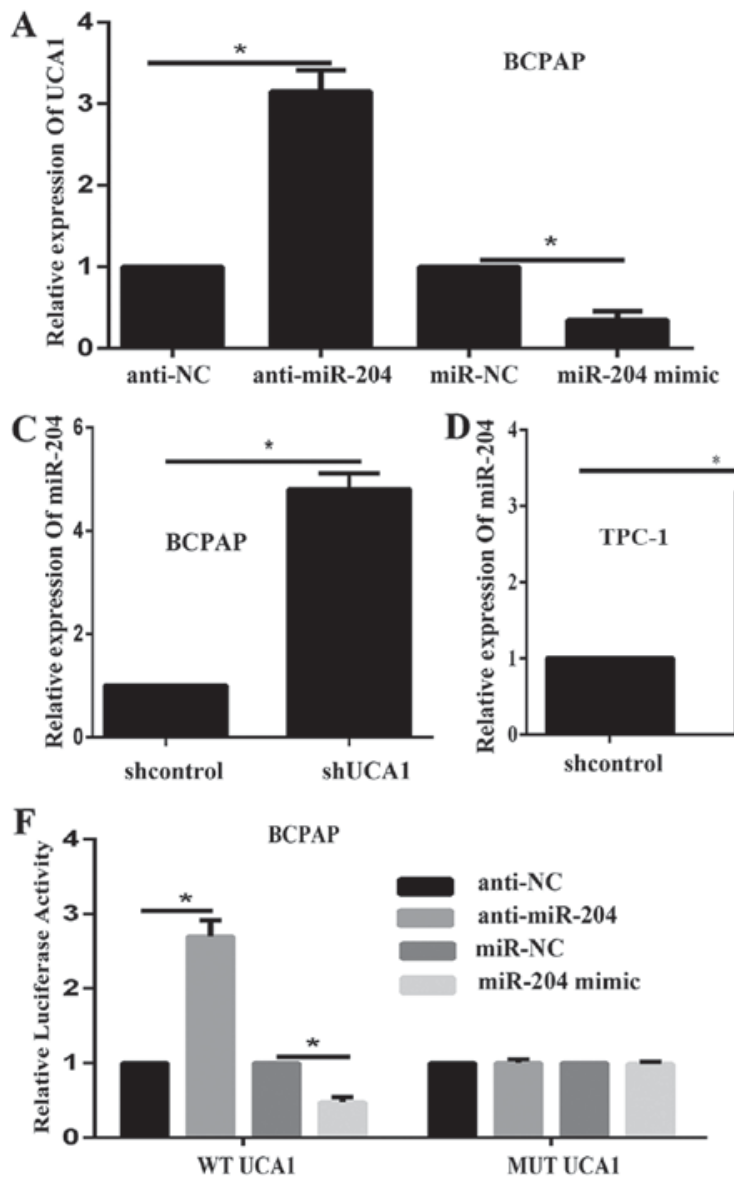

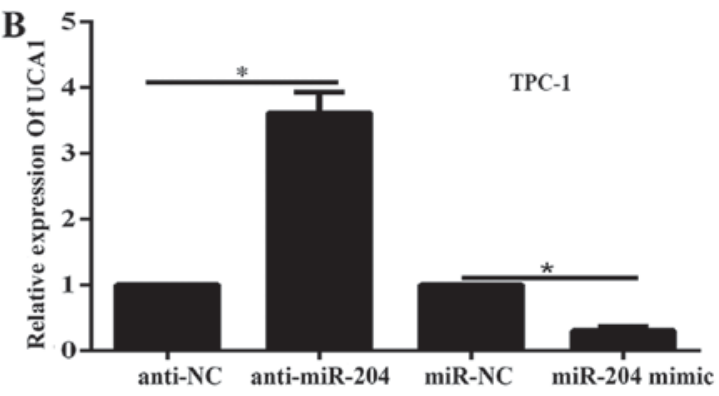

E 5' - ACACCUAUggGuUUCCCUA -3' MUT UCA 5' - ACACCUAUGGGAAAGGGUA - 3' WT UCA1 3'- GUAUCCUACUGUUUCCCUU -5' miR-204 5' - UUGCUUGUGCAAAAGGGAA -3' WT BRD4 5' - UUGCUUGUGCAUUUCCCUA -3' MUT BRD4 shUCA1

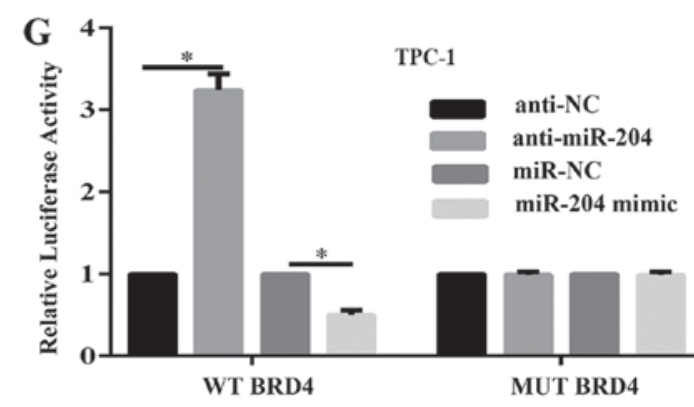

Figure 5. UCA1 competes with BRD4 for miR-204 binding. (A and B) The expression of UCA1 in response to miR-204 changes in (A) BCPAP and (B) TPC-1 cells was examined via RT-qPCR. (C and D) miR-204 expression in response to UCA1 silencing in (C) BCPAP and (D) TPC-1 cells was examined by RT-qPCR. (E) A WT or MUT UCA1 (wt-UCA1 and mut-UCA1 containing 6 bp mutation in the predicted binding sites of miR-204) or BRD4 3'UTR (wt-BRD4 3'UTR and mut-BRD4 3'UTR containing a 6 bp mutation in the predicted binding sites of miR-204) luciferase reporter gene vector was constructed. (F and G) Following overnight culture, cells were co-transfected with the indicated vectors and miR-204 mimics or miR-204 inhibitor, respectively. Luciferase assays were performed $48 \mathrm{~h}$ following transfection to determine the luciferase activity. "P $<0.05$, as indicated. UCA1, urothelial carcinoma-associated 1; sh-, short hairpin RNA; BRD4, bromodomain containing 4; miR, microRNA; NC, negative control; RT-qPCR, reverse transcription-quantitative polymerase chain reaction; WT, wild type; MUT, mutant; UTR, untranslated region.

in PTC. The expression level of UCA1 in response to miR-204 inhibition and overexpression in BCPAP and TPC-1 cell was examined via qPCR. Results showed that UCA1 was inhibited by miR-204 overexpression while enhanced by miR-204 inhibition (Fig. 5A and B). In addition, qPCR data illustrated that the expression of miR-204 was remarkably enhanced while UCA1 expression was downregulated (Fig. 5C and D). These results demonstrated a dual regulation between UCA1 and miR-204. According to online tools, UCA1 shared a same binding site in miR-204 with BRD4. Then luciferase assays were performed to discover the correlations between miR-204 and UCA1. Luciferase reporter gene vectors (wt-BRD4 3'UTR, mut-BRD4 3'UTR containing a 6 bp mutation on miR-204 binding site in the 3'UTR of BRD4, wt-UCA1, and mut-UCA1 containing a 6 bp mutation on miR-204 binding site in UCA1) were constructed and co-transfected into BCPAP and TPC-1 cells with miR-204 mimics or miR-204 inhibitor (Fig. 5E). The luciferase assays results showed that the luciferase activity of wt-UCA1 and wt-BRD4 vectors was remarkably decreased by miR-204 mimics, enhanced by miR-204 inhibitor; after mutation in the predicted binding sites of miR-204, the changes of the luciferase activity were abolished (Fig. 5F and G). These data suggested that UCA1 and BRD4 both could bind to miR-204. Given the consistency of the binding site(s), UCA1 might compete with BRD4 for miR-204 binding, so that to attenuate the inhibitory effect of miR-204 on BRD4 expression.

UCA1 regulated BRD4 expression via miR-204. To validate whether UCA1 regulated BRD4 expression through miR-204, we cotransfected BCPAP and TPC-1 cells with miR-204 inhibitor and shUCA1, and examined the expression of BRD4 by western blot and qPCR. The results showed that the expression of protein and mRNA of BRD4 were increased by miR-204 inhibition, decreased by shUCA1 (Fig. 3A-D). The promotive effect of miR-204 inhibition on the expression of BRD4 could be partially abolished by shUCA1 (Fig. 3A-D). These results demonstrated that UCA1 could regulate BRD4 expression through miR-204.

Knockdown of UCAl inhibits tumor growth in vivo. To further illustrate the effects of UCA1 on the PTC growth in vivo, we applied a xenograft model in which the BCPAP cells transfected with shUCA1 or shNC were subcutaneously 
A
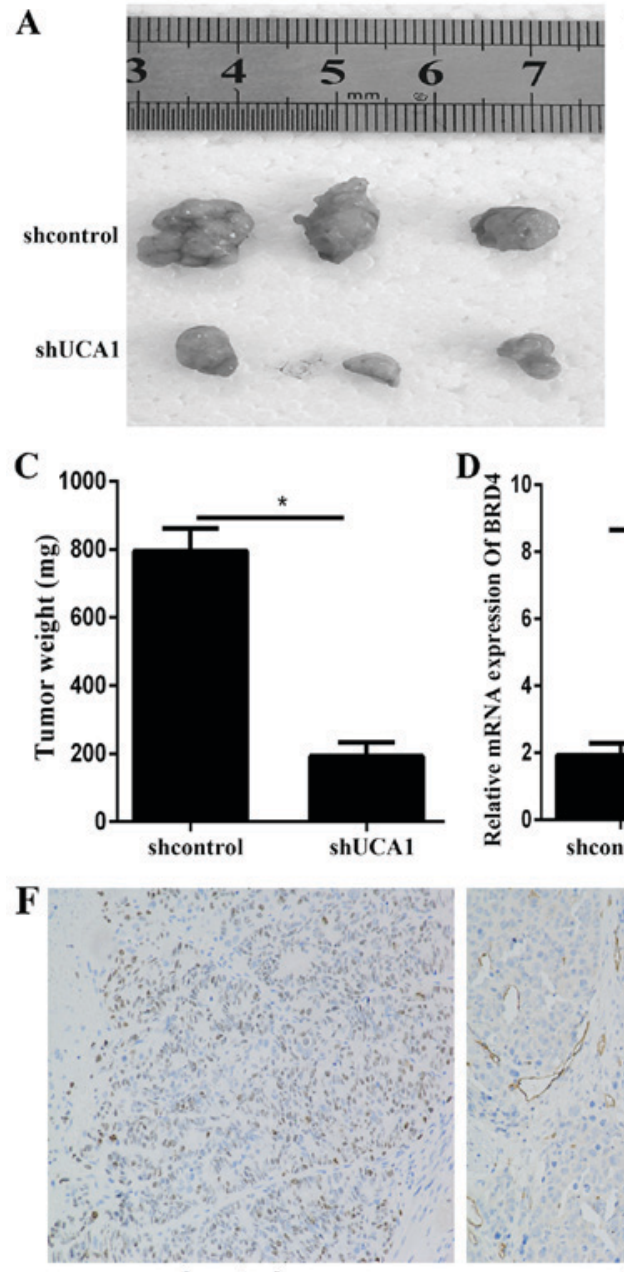

shcontrol
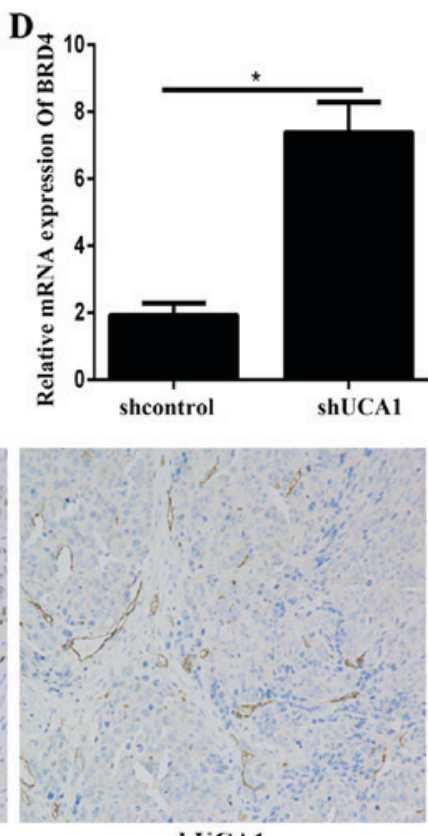
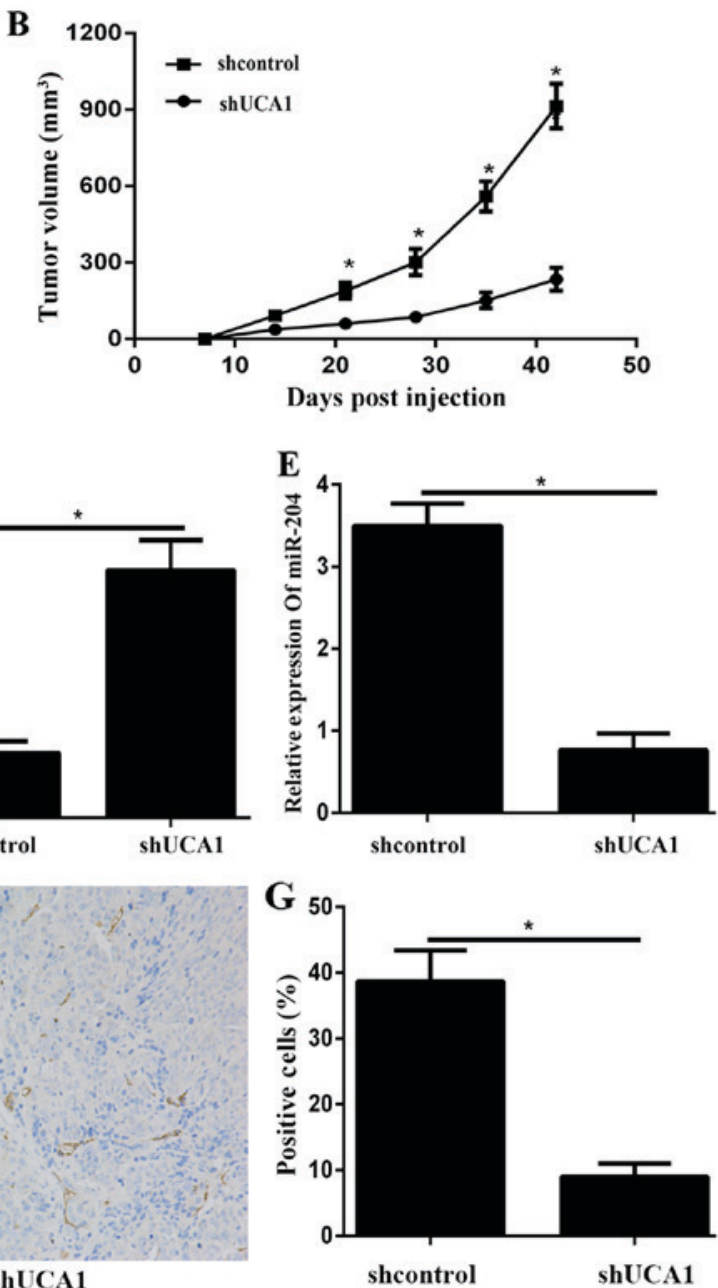

Figure 6. UCA1 silencing inhibits tumor growth in vivo. shUCA1 or shNC transfected BCPAP cells were injected subcutaneously into athymic nude mice ( $n=6 /$ group). (A) The shUCA1 tumor xenografts were smaller than the shNC tumor xenografts. (B) shUCA1 tumor growth was markedly slower than that observed in the shNC tumor xenografts following 42 days. (C) The average weight of the resected tumors. (D) RT-qPCR was used to detect the mRNA expression of BRD4. (E) RT-qPCR analysis of miR-204 expression levels in the shUCA1 and shNC tumor xenografts. (F) Representative images (magnification, $\mathrm{x} 200)$ of thyroid tumor sections from the shNC and shUCA1 tumors, stained for BRD4. (G) Quantification of the percentage of BRD4 (+) cells in the tumor sections. "P<0.05 vs. shcontrol/as indicated. UCA1, urothelial carcinoma-associated 1; sh-, short hairpin RNA; BRD4, bromodomain containing 4; miR, microRNA; NC, negative control; RT-qPCR, reverse transcription-quantitative polymerase chain reaction.

injected into the flanks of athymic mice and measured the tumor in a period time. The results showed that tumors formed by transfected shUCA1 grew more slowly than those formed by transfected shNC (Fig. 6A-C). Furthermore, qPCR results showed that the BRD4 expression levels in the shUCA1 tumour xenografts were lower than those in the control xenografts (Fig. 6D). In addition, the qPCR results showed that the expression level of miR-204 was significantly downregulated in shUCA1 transfected tumor compared to shNC transfected tumor (Fig. 6E). At last, histologic analysis showed that shUCA1 tumors had significantly fewer BRD4 positive cells than the shNC tumors (Fig. 6F and G). The in vivo results also demonstrated that UCA1 might regulate BRD4 via miR-204.

\section{Discussion}

In the present study, we confirmed the oncogenic role of UCA1 in the progression of PTC. Initially, we demonstrated that lncRNA UCA1 was significantly increased in PTC cell lines and tissues. Then, we showed that UCA1 silencing inhibited the viability, proliferation, migration and invasion of PTC cells in vitro and in vivo. Finally, we observed that UCA1 could sponge miR-204 and modulate BRD4 expression.

BRD4 is an epigenome reader and member of the bromodomain and extra-terminal family of proteins, which consist of two bromodomains in tandem and an extra-terminal domain. BRD4 promotes cell cycle progression, regulates cell growth and transcription and plays a critical role in the tumour progression of various cancers via different molecular mechanisms (21-28). BRD4 inhibition with JQ1 significantly prevents PTC progression in vitro and in vivo (20). The BRD4 expression in breast cancer is regulated by miR-599 (29). However, how BRD4 is aberrantly dysregulated in PTC is unclear, and the relation between miRNA and BRD4 has yet to be established. miR-204 plays a protective role by inhibiting thyroid cancer cell proliferation and may identify new targets for anti-cancer treatment (15). In the present study, luciferase assay and Western blot analysis confirmed that BRD4 was a direct target gene of miR-204. 
IncRNAs are transcribed RNA molecules with a length of more than 200 nucleotides that lack a substantial protein-coding potential. They can regulate protein-coding genes at epigenetic, transcriptional and post-transcriptional levels and participate in physiological processes. Various lncRNAs are frequently aberrantly expressed in cancers, and their differential expression is closely related to tumour progression; therefore, they serve as oncogenes or tumor suppressor genes (28). Further studies should be conducted to clarify the biological function and molecular mechanisms of lncRNAs in tumour progression.

UCA1 is an identified lncRNA that promotes tumor progression in a wide range of tumour types, including bladder cancer, breast cancer, hepatocellular carcinoma, colorectal cancer and gastric cancer (9,30-32). However, the role of UCA1 in PTC has yet to be illustrated. Initially, we investigated the mRNA expression of UCA1 in PTC tissues and cell lines and found that UCA1 expression was significantly higher in PTC tissues and cell lines than in normal tissues. Moreover, UCA1 levels in PTC tissues were positively correlated with advanced clinical stages and lymph node metastasis, but not with age, gender, or tumor size (data not shown). These data suggested that UCA1 functioned as an oncogene in PTC, and this finding is consistent with previous results on other tumors (9,30-32). To investigate the molecular mechanism by which UCA1 affects PTC proliferation and migration, we monitored the protein level of BRD4 when the UCA1 expression was downregulated by using shUCA1 in thyroid cancer. We showed that UCA1 silencing reduced the BRD4 protein expression, indicating the involvement of BRD4 in the regulation of UCA1 cell proliferation and invasion. A previous study demonstrated that lncRNAs act as competing endogenous RNAs to mediate miRNAs, and IncRNA-miRNA interaction regulates tumour progression through the regulation of oncogenes or tumour suppressor genes (33). UCA1 is related to miR-204 via the regulation of different downstream target genes (9-11). In the present study, we revealed the interaction between UCA1 and miR-204 in PTC for the first time; that is, UCA1 and miR-204 could negatively regulate each other. In this reciprocal negative regulation loop, the overexpression of UCA1 represses the expression of miR-204 to upregulate the expression of BRD4, thereby enhancing thyroid cancer cell growth and migration and invasion. On the other hand, the miR-204 represses the expression of UCA1 and decrease the expression of BRD4, so that to inhibit the tumor growth and metastasis. The equilibrium might depend on the tumour microenvironment and the type of cancer. We also showed that miR-204 could directly control the BRD4 expression. Given this result, we investigated whether miR-204 could regulate UCA1 and BRD4 through direct targeting. Dual luciferase assays revealed that UCA1 could bind to miR-204. miR-204 could also bind to the 3'UTR of BRD4, suggesting that UCA1 might compete with BRD4 for miR-204 binding to inhibit miR-204, to promote BRD4 expression and to affect PTC progression. To further discover whether BRD4 could regulate the expression of UCA1 and miR-204, we used siBRD4 to knockdown the expression of BRD4, and did not discover that BRD4 could regulate the expression of miR-204 and UCA1 in PTC cell lines (data not shown). These data demonstrated that BRD4 was a downstream target gene of
UCA1/miR-204 axis, and illustrated that drug resistance to BRD4 might select the upstream gene UCA1 or miR-204 as alternative therapy target.

In conclusion, the UCA1/miR-204/BRD4 axis plays a critical role in PTC cell proliferation and invasion and shows potential for therapeutic applications in patients with thyroid cancer.

\section{Acknowledgements}

Not applicable.

\section{Funding}

The present study was supported by the Foundation of Shandong People and Family Planning Commission (grant no. 2015WSA07008).

\section{Availability of data and materials}

All data generated or analyzed during this study are included in this published article.

\section{Authors' contributions}

DL and DH conceived and designed the experiments. CC, JC, $\mathrm{ZH}$ and $\mathrm{YW}$ conducted all of the experiments. YW wrote and revised the manuscript. All authors read and approved the final manuscript.

\section{Ethics approval and consent to participate}

The present study was approved by the Ethics Committee of Jining Medical University (Jining, China). All patients provided written informed consent.

\section{Patient consent for publication}

All patients provided written informed consent for the publication of any associated data and accompanying images.

\section{Competing interests}

The authors declare that they have no competing interests.

\section{References}

1. Maniakas A, Davies L and Zafereo ME: Thyroid disease around the World. Otolaryngol Clin North Am 51: 631-642, 2018

2. Siegel RL, Miller KD and Jemal A: Cancer statistics, 2017. CA Cancer J Clin 67: 7-30, 2017.

3. Xing M: Molecular pathogenesis and mechanisms of thyroid cancer. Nat Rev Cancer 13: 184-199, 2013.

4. Lupoli R, Cacciapuoti M, Tortora A, Barba L, Verde N, Romano F, Vastarella M, Fonderico F, Masone S, Milone M, et al: Clinical outcome in differentiated thyroid carcinoma and microcarcinoma. Int J Surg 12 (Suppl 1): S148-S151, 2014.

5. Mercer TR and Mattick JS: Structure and function of long noncoding RNAs in epigenetic regulation. Nat Struct Mol Biol 20: 300-307, 2013.

6. Lin CY and Xu HM: Novel perspectives of long non-coding RNAs in esophageal carcinoma. Carcinogenesis 36: 1255-1262, 2015.

7. Huarte M: The emerging role of lncRNAs in cancer. Nat Med 21: 1253-1261, 2015. 
8. Xie $\mathrm{H}, \mathrm{Ma} \mathrm{H}$ and Zhou D: Plasma HULC as a promising novel biomarker for the detection of hepatocellular carcinoma. Biomed Res Int 2013: 136106, 2013.

9. Zhang S, Dong X, Ji T, Chen G and Shan L: Long non-coding RNA UCA1 promotes cell progression by acting as a competing endogenous RNA of ATF2 in prostate cancer. Am J Transl Res 9: 366-375, 2017.

10. Wang X, Yang B and Ma B: The UCA1/miR-204/Sirt1 axis modulates docetaxel sensitivity of prostate cancer cells. Cancer Chemother Pharmacol 78: 1025-1031, 2016.

11. Jiao C, Song Z, Chen J, Zhong J, Cai W, Tian S, Chen S, Yi Y and Xiao Y: IncRNA-UCA1 enhances cell proliferation through functioning as a ceRNA of Sox4 in esophageal cancer. Oncol Rep 36: 2960-2966, 2016.

12. Wang ZQ, Cai Q, Hu L, He CY, Li JF, Quan ZW, Liu BY, Li C and Zhu ZG: Long noncoding RNA UCA1 induced by SP1 promotes cell proliferation via recruiting EZH2 and activating AKT pathway in gastric cancer. Cell Death Dis 8: e2839, 2017.

13. Wang X, Gao Z, Liao J, Shang M, Li X, Yin L, Pu Y and Liu R lncRNA UCA1 inhibits esophageal squamous-cell carcinoma growth by regulating the Wnt signaling pathway. J Toxicol Environ Health A 79: 407-418, 2016.

14. Wang F, Zhou J, Xie X, Hu J, Chen L, Hu Q, Guo H and Yu C: Involvement of SRPK1 in cisplatin resistance related to long non-coding RNA UCA1 in human ovarian cancer cells Neoplasma 62: 432-438, 2015

15. Wu ZY, Wang SM, Chen ZH, Huv SX, Huang K, Huang BJ, Du JL, Huang CM, Peng L, Jian ZX and Zhao G: MiR-204 regulates HMGA2 expression and inhibits cell proliferation in human thyroid cancer. Cancer Biomark 15: 535-542, 2015.

16. Liu L, Wang J, Li X, Ma J, Shi C, Zhu H, Xi Q, Zhang J, Zhao X and Gu M: MiR-204-5p suppresses cell proliferation by inhibiting IGFBP5 in papillary thyroid carcinoma. Biochem Biophys Res Commun 457: 621-626, 2015.

17. Bian Z, Jin L, Zhang J, Yin Y, Quan C, Hu Y, Feng Y, Liu H, Fei B, Mao Y, et al: LncRNA-UCA1 enhances cell proliferation and 5-fluorouracil resistance in colorectal cancer by inhibiting miR-204-5p. Sci Rep 6: 23892, 2016.

18. Saiselet M, Floor S, Tarabichi M, Dom G, Hébrant A, van Staveren WC and Maenhaut C: Thyroid cancer cell lines: An overview. Front Endocrinol (Lausanne) 3: 133, 2012.

19. Livak KJ and Schmittgen TD: Analysis of relative gene expression data using real-time quantitative PCR and the 2(-Delta Delta C(T)) method. Methods 25: 402-408, 2001

20. Gao X, Wu X, Zhang X, Hua W, Zhang Y, Maimaiti Y, Gao Z and Zhang Y: Inhibition of BRD4 suppresses tumor growth and enhances iodine uptake in thyroid cancer. Biochem Biophys Res Commun 469: 679-685, 2016.

21. Wang YH, Sui XM, Sui YN, Zhu QW, Yan K, Wang LS, Wang F and Zhou JH: BRD4 induces cell migration and invasion in HCC cells through MMP-2 and MMP-9 activation mediated by the Sonic hedgehog signaling pathway. Oncol Lett 10: 2227-2232, 2015 .
22. Segura MF, Fontanals-Cirera B, Gaziel-Sovran A, Guijarro MV, Hanniford D, Zhang G, González-Gomez P, Morante M, Jubierre L, Zhang W, et al: BRD4 sustains melanoma proliferation and represents a new target for epigenetic therapy. Cancer Res 73: 6264-6276, 2013.

23. Puissant A, Frumm SM, Alexe G, Bassil CF, Qi J, Chanthery YH, Nekritz EA, Zeid R, Gustafson WC, Greninger P, et al: Targeting MYCN in neuroblastoma by BET bromodomain inhibition. Cancer Discov 3: 308-323, 2013.

24. Patel AJ, Liao CP, Chen Z, Liu C, Wang Y and Le LQ: BET bromodomain inhibition triggers apoptosis of NF1-associated malignant peripheral nerve sheath tumors through Bim induction. Cell Rep 6: 81-92, 2014.

25. Ott CJ, Kopp N, Bird L, Paranal RM, Qi J, Bowman T, Rodig SJ, Kung AL, Bradner JE and Weinstock DM: BET bromodomain inhibition targets both c-Myc and IL7R in high-risk acute lymphoblastic leukemia. Blood 120: 2843-2852, 2012.

26. Lockwood WW, Zejnullahu K, Bradner JE and Varmus $\mathrm{H}$ : Sensitivity of human lung adenocarcinoma cell lines to targeted inhibition of BET epigenetic signaling proteins. Proc Natl Acad Sci USA 109: 19408-19413, 2012.

27. Andrieu G, Tran AH, Strissel KJ and Denis GV: BRD4 regulates breast cancer dissemination through Jagged1/Notch1 signaling. Cancer Res 76: 6555-6567, 2016.

28. He Y, Meng XM, Huang C, Wu BM, Zhang L, Lv XW and Li J: Long noncoding RNAs: Novel insights into hepatocelluar carcinoma. Cancer Lett 344: 20-27, 2014.

29. Wang Y, Sui Y, Zhu Q and Sui X: Hsa-miR-599 suppresses the migration and invasion by targeting BRD4 in breast cancer. Oncol Lett 14: 3455-3462, 2017.

30. Wang F, Ying HQ, He BS, Pan YQ, Deng QW, Sun HL, Chen J, Liu X and Wang SK: Upregulated IncRNA-UCA1 contributes to progression of hepatocellular carcinoma through inhibition of miR-216b and activation of FGFR1/ERK signaling pathway. Oncotarget 6: 7899-7917, 2015.

31. Zheng Q, Wu F, Dai WY, Zheng DC, Zheng C, Ye H, Zhou B, Chen JJ and Chen P: Aberrant expression of UCA1 in gastric cancer and its clinical significance. Clin Transl Oncol 17: 640-646, 2015

32. Liu M, Xing LQ and Liu YJ: A three-long noncoding RNA signature as a diagnostic biomarker for differentiating between triple-negative and non-triple-negative breast cancers. Medicine (Baltimore) 96: e6222, 2017.

33. Li JH, Zhang SQ, Qiu XG, Zhang SJ, Zheng SH and Zhang DH: Long non-coding RNA NEAT1 promotes malignant progression of thyroid carcinoma by regulating miRNA-214. Int J Oncol 50: 708-716, 2017. 